\title{
Avaliação do risco epidemiológico e do desempenho dos programas de controle de tuberculose nas Regiões de Saúde do estado de Santa Catarina, 2003 a 2010*
}

\author{
Evaluation of epidemiologic risk and Tuberculosis Control Program performance by health \\ regions, State of Santa Catarina, Brazil, 2003-2010
}

\author{
Sergio Adam Mendonça \\ Fundação Universidade Regional de Blumenau, Departamento de Medicina, Blumenau-SC, Brasil \\ Selma Cristina Franco \\ Universidade da Região de Joinville, Departamento de Medicina, Joinville-SC, Brasil
}

\section{Resumo}

Objetivo: avaliar o risco epidemiológico e o desempenho dos programas de controle de tuberculose segundo Regiões de Saúde do estado de Santa Catarina, Brasil, no período de 2003 a 2010. Métodos: estudo ecológico com dados fornecidos pela vigilância epidemiológica estadual, compreendendo 4 indicadores de risco e 11 de desempenho do programa; calculou-se a mediana de cada indicador por Região e atribuiu-se pontos conforme a distribuição percentilar, permitindo-se a criação de um índice de gravidade. Resultados: verificou-se a existência de diferentes níveis de risco de morbimortalidade e distintos índices de desempenho das ações de controle da tuberculose nas Regiões de Saúde; 0 índice de gravidade permitiu identificar 6 Regiões de maior gravidade, 9 intermediárias e 6 de menor gravidade, com diferenças nos escores de risco e desempenho $(\mathrm{p}<0,001)$. Conclusão: evidenciou-se grande variedade de nichos de gravidade distribuídos nas diversas Regiões, bem como diversidade no empenho da gestão administrativa para seu enfrentamento.

Palavras-chave: Tuberculose; Avaliação de Programas e Projetos de Saúde; Regionalização; Planos e Programas de Saúde.

\begin{abstract}
Objective: to evaluate epidemiologic risk and Tuberculosis Control Program performance by Health Region in Santa Catarina State, Brazil, 2003-2010. Methods: this was an ecological study using state epidemiologic surveillance data comprising 4 risk indicators and 11 Tuberculosis Program performance indicators; we calculated the median for each indicator by region and gave scores according to percentile ranking. A severity index was thus created. Results: different levels of morbi-mortality risk and distinct Tuberculosis control performance indexes were found in the regions; the severity index created allowed us to identify six regions with high severity, nine intermediate and six with low severity, although there were differences in risk and performance scores $(p<0.001)$. Conclusion: a great variety was found in the distribution of pockets of disease severity in the different regions, as was diversity in health management efforts to overcome them.
\end{abstract}

Key words: Tuberculosis; Program Evaluation; Regional Health Planning; Health Programs and Plans.

\footnotetext{
* O presente artigo resultou de pesquisa de dissertação de Mestrado no âmbito do Programa de Pós-Graduação em Saúde e Meio Ambiente da Universidade da Região de Joinville (Univille), defendida em 2013 por Sergio Adam Mendonça.
} 


\section{Introdução}

A tuberculose (TB) é um grave problema de Saúde Pública no Brasil e no mundo, sendo a segunda maior causa de morte por doença infecciosa, apenas superada pelas mortes associadas ao vírus da imunodeficiência humana (HIV) ${ }^{1,2}$ Além da magnitude, a doença possui um importante componente social e tende a afligir populações mais fragilizadas. ${ }^{2}$ Segundo a Organização Mundial da Saúde (OMS), em 2011, ocorreram no mundo 9 milhões de casos novos de tuberculose e 1,4 milhões de mortes pela doença. ${ }^{3}$ Atualmente, o Brasil ocupa $017^{\circ}$ lugar entre os 22 países responsáveis por $82 \%$ do total de casos de tuberculose no mundo, ${ }^{3}$ sendo preocupante o fato de a OMS afirmar que o país detectou somente 91\% de seus casos novos em 2010, totalizando 70 mil casos novos. Além disso, no Brasil, morrem a cada ano cerca de 4.600 pessoas por causa da tuberculose. ${ }^{2}$ Sob diversos aspectos, a tuberculose ainda é considerada uma doença negligenciada, seja por subnotificação e obstáculos para o acesso universal ao diagnóstico e tratamento, seja pela falta de compreensão da dimensão da doença dentro da comunidade e, inclusive, entre profissionais e gestores da Saúde. ${ }^{4}$

Diante dos desafios impostos pela carga da doença no Brasil, o Programa Nacional de Controle da Tuberculose (PNCT) do Ministério da Saúde orienta que suas estratégias sejam desenvolvidas em três níveis de gestão: federal, estadual e municipal. ${ }^{5}$ Em cada um desses níveis, dever-se-ão desenvolver atividades específicas, cabendo à esfera estadual as ações de monitoramento e avaliação dos programas municipais agrupados em Regiões de Saúde; e ao município, as ações de prevenção, diagnóstico e tratamento, preferencialmente junto à atenção básica. ${ }^{5}$

\section{A tuberculose ainda é considerada uma doença negligenciada, seja por subnotificação e obstáculos para o acesso universal ao diagnóstico e tratamento, seja pela falta de compreensão da dimensão da doença.}

A avaliação dos programas de tuberculose é feita a partir da análise de 21 indicadores de saúde recomendados pelo Ministério. ${ }^{5}$ Os indicadores de impacto mostram a situação epidemiológica com relação à doença e necessitam de um tempo maior para serem modificados, além de refletirem a influência de fatores externos às atividades de controle da doença. 0 s indicadores de resultado medem o desempenho dos programas, podendo ser avaliados no médio prazo. ${ }^{5}$

Em Santa Catarina, historicamente, as taxas de incidência da doença têm se mantido entre as mais baixas dos estados da federação: 27,8 por 100 mil habitantes em 2008. Segundo dados do próprio Ministério da Saúde, o estado apresenta uma situação epidemiológica regular com incidência moderada a baixa e, em geral, boa qualidade de vigilância. ${ }^{6,7}$ Verifica-se, entretanto, persistência da doença e uma variabilidade nos indicadores de ocorrência e mortalidade em Santa Catarina.

0 presente estudo teve como objetivo avaliar o risco epidemiológico e o desempenho dos programas de controle de tuberculose segundo as Regiões de Saúde do estado de Santa Catarina, no período de 2003 a 2010.

\section{Métodos}

Trata-se de estudo de delineamento ecológico transversal, tendo como unidades de análise as 21 Regiões de Saúde do estado de Santa Catarina, onde são operacionalizadas as ações do Programa de Controle da Tuberculose. ${ }^{8}$ Os anos de 2003 a 2010 foram selecionados pelo fato de disporem de registros dos casos notificados de forma padronizada e contínua. Os dados foram obtidos junto à vigilância epidemiológica estadual, responsável pela compilação sistemática dos casos registrados no Sistema de Informação de Agravos de Notificação (Sinan).

No período estudado, a população de Santa Catarina cresceu de 5,6 milhões (2003) para 6,2 milhões (2010) de habitantes, distribuídos em 293 municípios, por sua vez agrupados em 21 Regiões de Saúde definidas seguindo uma lógica de organização de serviços de saúde e fluxos assistenciais, conforme estabelece o Plano Diretor de Regionalização (PDR) vigente. ${ }^{8}$

Entre os indicadores recomendados pelo Ministério da Saúde, ${ }^{5}$ selecionaram 4 indicadores de impacto, capazes de refletir o quadro epidemiológico da doença. Três desses indicadores - taxa de incidência de tuberculose, taxa de incidência de tuberculose pulmonar bacilifera e taxa de mortalidade - são marcadores populacionais tradicionais da doença, guardam íntima correlação com o nível de disseminação da tuberculose (TB) e a gravidade da situação dentro da comunidade. 
São indicadores da transcendência e magnitude da doença. 0 quarto indicador - coinfecção TB/HIV - foi utilizado neste trabalho como representante indireto da incidência de infectados pelo HIV nas referidas Regiões. ${ }^{9}$

Também em conformidade com as determinações do Ministério da Saúde, selecionaram-se mais 11 indicadores dos diferentes aspectos do desempenho do programa, a exemplo do diagnóstico bacteriológico, do tratamento e da prevenção. ${ }^{5}$ São os chamados de indicadores de resultado:

- proporção de contatos de casos de tuberculose examinados entre os registrados;

- proporção de casos novos de tuberculose testados para o HIV;

- proporção de casos de tuberculose curados;

- proporção de casos curados com comprovação bacteriológica;

- proporção de abandono entre os casos novos;

- proporção de casos encerrados com óbito (letalidade);

- proporção de casos de tuberculose que realizaram tratamento diretamente observado (TDO);

- proporção de casos novos que realizaram baciloscopia;

- proporção de casos novos confirmados bacteriologicamente;

- proporção de casos de retratamento que realizaram cultura;

- proporção de casos de retratamento; e

- proporção de casos de tuberculose encerrados., ${ }^{5,6}$

De acordo com os valores alcançados por esses dois grupos de indicadores, elaborou-se um panorama da situação epidemiológica e do desempenho dos programas de controle da tuberculose nas 21 Regiões de Saúde do estado.

Inicialmente, os quatro indicadores de impacto foram utilizados para determinar o risco e a gravidade da doença em cada Região. Para tanto, utilizou-se a mediana de cada indicador no período entre 2003 e 2010, para cada Região de Saúde. As medianas foram ordenadas e cada indicador classificado, conforme seu percentil, em níveis de risco: baixo risco (abaixo do percentil 25), risco intermediário (entre os percentis 25 e 75) e risco elevado (acima do percentil 75). Na etapa seguinte, foram atribuídos pontos para quantificar os diversos riscos em cada Região: um ponto para indicador de risco baixo, dois para risco intermediário ou três pontos para risco elevado. Ao se adotar essa sistemática, considerando-se os quatro indicadores de impacto, cada Região de Saúde poderia receber pontuação mínima de quatro e máxima de 12 pontos. Assim, de acordo com o risco epidemiológico, as Regiões de Saúde foram então classificadas como de baixo risco (4 a 6 pontos), de risco intermediário ( 7 a 9 pontos) ou de alto risco (10 a 12 pontos). Partiu-se da premissa de que as populações das Regiões onde foram obtidas as maiores pontuações não só estariam sob maior risco de adoecimento como apresentariam formas mais graves da doença, enquanto para as Regiões com as menores pontuações, a situação seria inversa.

Utilizando-se a mesma metodologia, foi elaborado um escore dos indicadores de resultado com o objetivo de medir o desempenho das atividades do programa implantadas nas Regiões de Saúde. Para tanto, calculou-se a mediana dos 11 indicadores no período, para cada Região, com a ressalva de que nos quesitos referentes a abandono, óbito e retratamento, quanto menores as proporções correspondentes, melhor seria a situação da população frente à tuberculose; nestes casos, a pontuação foi invertida. Utilizou-se o mesmo procedimento para os indicadores de risco, classificando-se as Regiões como de desempenho ruim (1 ponto), intermediário ( 2 pontos) ou bom (3 pontos). Cada Região de Saúde poderia obter o mínimo de 11 pontos e o máximo de 33 pontos. Assim, as Regiões de Saúde foram classificadas como de desempenho bom ( 26 a 33 pontos), intermediário (21 a 25 pontos) ou ruim (11 a 20 pontos).

Considerando-se que não há uma correlação de importância ou gravidade para cada um dos indicadores, estes foram pontuados igualitariamente. Afinal, a proposta do estudo foi medir o número de vezes em que cada Região foi classificada como boa, intermediária ou ruim, tanto no risco como no desempenho.

Para comparar os três grupos de risco e de desempenho das Regiões, utilizou-se o teste de Kruskal Wallis; e para verificar as diferenças dos grupos dois a dois, o teste Dunn, de comparação múltipla. 0 resultado deste segundo teste foi apresentado na forma de letras, ' $a$ ' ou 'b', sendo que letras diferentes representaram diferenças significativas. Ou seja, ' $a$ ' foi diferente de 'b', porém 'ab' não foi significativamente diferente de 'a' e tampouco de 'b'. Considerou-se o nível de significância estatística de 5\%. 
Finalmente, propôs-se a criação de um índice de gravidade para cada Região de Saúde baseado na razão entre os escores de risco e de desempenho, uma vez que existe uma correlação inversa entre os escores obtidos para os indicadores de risco (quanto menor, melhor) e desempenho (quanto maior, melhor). Ao se considerar a melhor situação definida pelos indicadores de risco - 4 pontos - e o melhor desempenho - 33 pontos -, ponderou-se a razão entre estes dois grupos de indicadores como 0,879 , equivalente à nota 10. Para modificar a direção e melhor compreender a disposição dos índices, desenvolveu-se uma fórmula que melhor os expressasse: 1 menos a razão entre 0 escore de indicadores, visto que apresentados dessa forma, obter-se-ia o pior desempenho com o índice menor e o melhor desempenho com o índice maior.

0 presente estudo, por haver trabalhado exclusivamente com dados secundários de acesso público, sem identificação dos indivíduos-casos, foi dispensado de apreciação por Comitê de Ética, em conformidade com a Resolução do Conselho Nacional de Saúde (CNS) $n^{0}$ 466, de 12 de dezembro de 2012.

\section{Resultados}

A classificação de risco epidemiológico a partir dos indicadores de impacto evidenciou 6 Regiões de Saúde com baixo risco, 9 com risco intermediário e $6 \mathrm{com}$ risco elevado. Constataram-se grandes variações nos valores alcançados para cada indicador, entre as Regiões. No período 2003-2010, as Regiões consideradas de maior risco epidemiológico tiveram pontuação entre 10 e 12, as de risco intermediário entre 7 e 9 , e as de baixo risco entre 4 e 6 . A comparação dos indicadores mostrou diferença significativa $(\mathrm{p}<0,001)$ entre as Regiões de baixo e as de elevado risco (Tabelas 1 e 2).

\section{Tabela 1 - Classificação das Regiões de Saúde segundo o risco epidemiológico de tuberculose no estado de} Santa Catarina, 2003 a 2010

\begin{tabular}{|c|c|c|c|c|c|}
\hline Regiões de Saúde & $\begin{array}{c}\text { Taxa de incidência } \\
\text { de tuberculose } \\
\text { (por } 100 \text { mil } \\
\text { habitantes) }\end{array}$ & $\begin{array}{c}\text { Taxa de incidência de } \\
\text { tuberculose pulmonar } \\
\text { bacilífera }\end{array}$ & $\begin{array}{c}\text { Taxa de } \\
\text { mortalidade por } \\
\text { tuberculose } \\
\text { (por } 100 \text { mil } \\
\text { habitantes) }\end{array}$ & $\begin{array}{c}\text { Proporção de } \\
\text { coinfecção TB/ } \\
\text { HIV } \\
\text { (\%) }\end{array}$ & $\begin{array}{l}\text { Escore } \\
\text { geral }^{\mathbf{a}}\end{array}$ \\
\hline São Miguel do Oeste & 5,0 & 3,5 & 0,2 & - & 4 \\
\hline Chapecó & 8,9 & 6,2 & 0,2 & 5,0 & 4 \\
\hline Xanxerê & 10,3 & 6,6 & 0,5 & 9,8 & 7 \\
\hline Concórdia & 6,0 & 2,1 & 0,4 & - & 4 \\
\hline Joaçaba & 13,3 & 7,7 & 0,6 & 6,5 & 6 \\
\hline Videira & 14,8 & 8,6 & 0,9 & 10,0 & 8 \\
\hline Rio do Sul & 8,0 & 4,4 & 0,2 & 12,2 & 5 \\
\hline Timbó & 14,2 & 6,4 & 0,4 & 11,5 & 6 \\
\hline Blumenau & 21,8 & 13,2 & 0,4 & 18,8 & 8 \\
\hline Itajaí & 80,7 & 40,8 & 1,8 & 24,4 & 12 \\
\hline Balneário de Camboriú & 51,0 & 20,8 & 1,1 & 22,7 & 11 \\
\hline Brusque & 25,4 & 11,6 & 1,8 & 6,3 & 9 \\
\hline Grande Florianópolis & 36,6 & 19,5 & 1,0 & 29,1 & 11 \\
\hline Tubarão & 24,7 & 10,6 & 1,2 & 23,4 & 10 \\
\hline Criciúma & 30,3 & 18,2 & 0,9 & 21,2 & 11 \\
\hline Araranguá & 16,7 & 12,0 & 1,1 & 13,9 & 8 \\
\hline Joinville & 40,0 & 19,0 & 1,3 & 16,3 & 11 \\
\hline Jaraguá do Sul & 19,4 & 9,2 & 0,5 & 8,7 & 8 \\
\hline Mafra & 16,0 & 7,6 & 1,3 & 6,2 & 8 \\
\hline Canoinhas & 19,8 & 9,8 & 1,6 & 9,0 & 9 \\
\hline Lages & 15,5 & 4,4 & 0,8 & 29,9 & 8 \\
\hline
\end{tabular}

a) $\square$ Escore baixo=4 a 6 pontos; $\square$ Escore intermediário = 7 a 9 pontos; $\square$ Escore elevado = 10 a 12 pontos 
Tabela 2 - Classificação da mediana dos indicadores de risco epidemiológico dos programas de controle da tuberculose no estado de Santa Catarina, 2003 a 2010

\begin{tabular}{|c|c|c|c|c|c|}
\hline $\begin{array}{l}\text { Classificação dos } \\
\text { indicadores }\end{array}$ & $\begin{array}{l}\text { Taxa de incidência } \\
\text { de tuberculose } \\
\text { (por } 100 \text { mil } \\
\text { habitantes) }\end{array}$ & $\begin{array}{c}\text { Taxa de incidência de } \\
\text { tuberculose pulmonar } \\
\text { bacilífera } \\
\text { (por } 100 \text { mil habitantes) }\end{array}$ & $\begin{array}{c}\text { Taxa de mortalidade } \\
\text { por tuberculose } \\
(\%)\end{array}$ & $\begin{array}{c}\text { Proporção de } \\
\text { coinfecção TB/ } \\
\text { HIV }\end{array}$ & $\begin{array}{l}\text { Escore } \\
\text { geral * }\end{array}$ \\
\hline & \multicolumn{5}{|c|}{ Mediana } \\
\hline Baixo & $8,4^{\mathrm{a}}$ & $4,4^{\mathrm{a}}$ & $0,3^{\mathrm{a}}$ & $5,6^{\mathrm{a}}$ & $4,5^{\mathrm{a}}$ \\
\hline Intermediário & $16,7^{\mathrm{ab}}$ & $9,2^{\mathrm{ab}}$ & $0,9^{\mathrm{ab}}$ & $11,5^{\mathrm{ab}}$ & $8^{\mathrm{ab}}$ \\
\hline Elevado & $38,3^{\mathrm{b}}$ & $19,2^{b}$ & $1,4^{b}$ & $23,9^{b}$ & $11^{b}$ \\
\hline
\end{tabular}

*Escore baixo $=4$ a 6 pontos; Escore intermediário $=7$ a 9 pontos; Escore elevado $=10$ a 12 pontos

Notas:

Todas as diferencas foram estatisticamente significativas conforme 0 Teste Kruskal Wallis $(p<0,001)$

As letras ' $a$ ', $b$ ' $e^{\prime} a b^{\prime}$ foram usadas para representar os resultados do teste de Dunn, que compara os grupos dois a dois. As letras diferentes ('a' $e^{\prime} b^{\prime}$ ) mostram diferenças significativas entre os grupos comparados e as letras que se repetem ('a' $\mathrm{e}^{\prime} a b^{\prime} \mathrm{o} \mathrm{u}^{\prime} \mathrm{b}^{\prime} \mathrm{e}^{\prime} a b^{\prime}$ ') mostram que as diferenças não foram significativas segundo 0 teste de Dunn.

A avaliação de desempenho dos programas de controle de tuberculose a partir de indicadores de resultado evidenciou 6 Regiões de desempenho ruim, 9 de desempenho intermediário e 6 de desempenho bom. Novamente, observou-se grande variabilidade entre os índices alcançados pela maioria dos indicadores.

As Regiões de Saúde consideradas como de desempenho bom tiveram pontuação entre 26 e 32 , as de desempenho intermediário entre 21 e 25 , e as de desempenho ruim entre 14 e 19. A comparação dos indicadores mostrou diferença significativa $(\mathrm{p}<0,001)$ entre as Regiões de desempenho bom e as de desempenho ruim (Tabela 3 e Tabela 4).

Finalmente, a distribuição das Regiões de Saúde segundo o índice de gravidade proposto mostrou que as Regiões de Chapecó e São Miguel do Oeste se encontravam em melhor situação, e as Regiões de Balneário Camboriú e Grande Florianópolis em pior situação frente ao risco epidemiológico e o desempenho de seus programas de controle da tuberculose (Tabela 5).

\section{Discussão}

0 desenho ecológico do presente estudo possibilitou mostrar grandes variações quanto ao risco epidemiológico para a tuberculose e o desempenho dos programas de controle da doença nas 21 Regiões de Saúde do estado de Santa Catarina. 0 índice de gravi- dade proposto permitiu identificar 6 Regiões de maior gravidade, 9 de gravidade intermediária e 6 de menor gravidade, com diferenças significativas nos escores de risco e de desempenho $(\mathrm{p}<0,001)$. Evidencia-se um potencial de melhoria passível de ser alcançado, no sentido de aprimorar o controle da doença.

De modo geral, a situação do estado, quando comparada à do resto do Brasil, é satisfatória, especialmente quanto aos indicadores principais da doença, como incidência e mortalidade. ${ }^{7,9,10}$ Porém, quando as Regiões de Saúde são analisadas isoladamente e comparadas umas às outras, percebe-se uma grande variedade de nichos de gravidade distribuídos entre diversas delas, assim como distintos empenhos da gestão administrativa para seu enfrentamento.

Embora o delineamento ecológico não possibilite afirmar que aquilo que ocorre em nível regional se reproduza, igualmente, em cada município, os achados deste estudo evidenciaram situações potencialmente mais graves, com maior risco de infecção e pior desempenho do programa de controle da tuberculose. É o caso de Itajaí, Balneário de Camboriú, Grande Florianópolis e Tubarão. Joinville e Criciúma, também com pontuação elevada no escore de risco, tiveram, em contrapartida, desempenho elevado, contrabalançando positivamente a situação de gravidade. Outra situação foi verificada em Lages e Canoinhas, ambas com risco intermediário, porém com desempenho ruim do programa. 


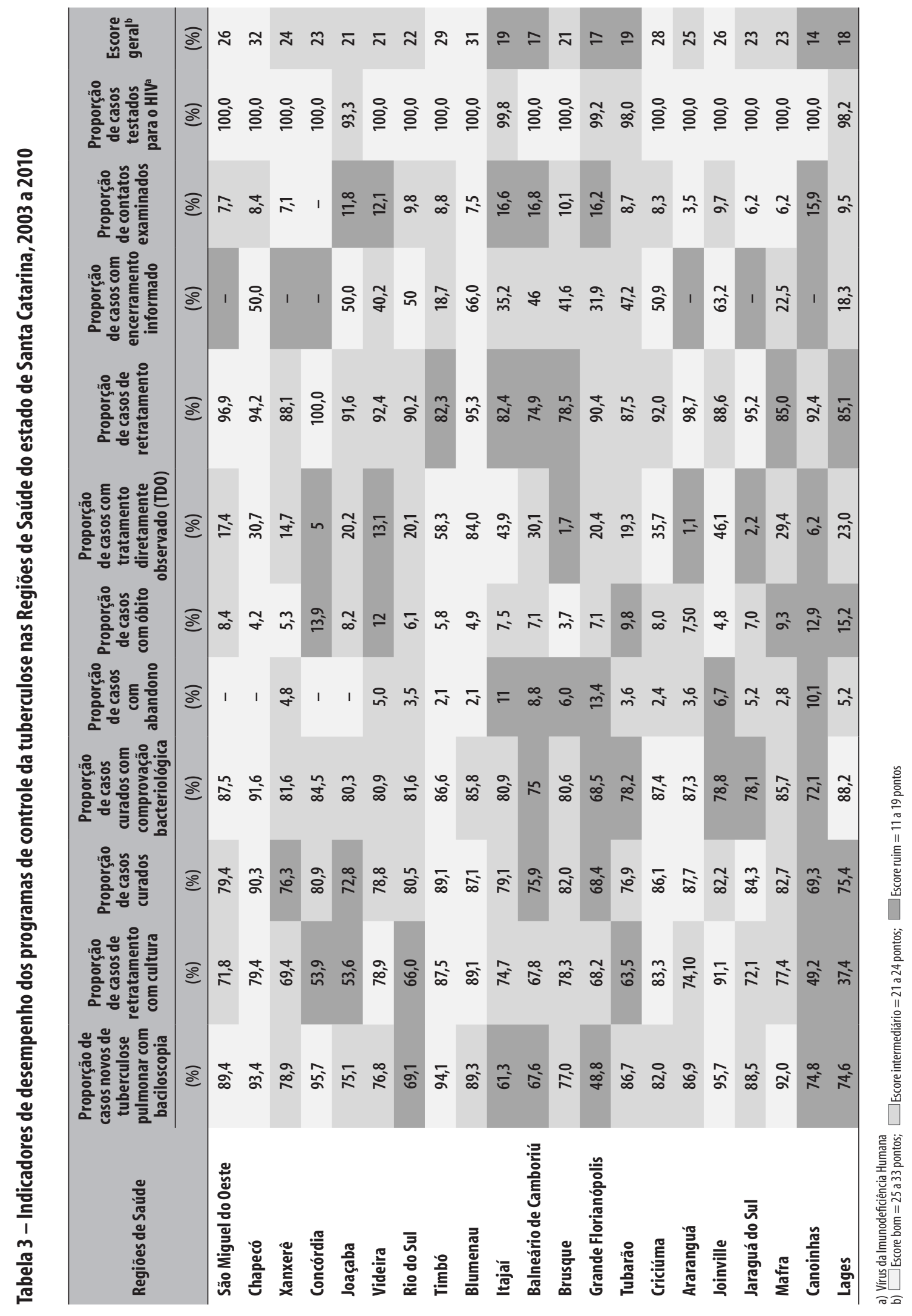


Tabela 4 - Classificação da mediana dos indicadores de desempenho dos programas de controle da tuberculose no estado de Santa Catarina, 2003 a 2010

\begin{tabular}{|c|c|c|c|c|c|c|}
\hline \multirow[t]{2}{*}{ Classificação dos indicadores } & $\begin{array}{l}\text { Proporção de } \\
\text { casos novos de } \\
\text { tuberculose } \\
\text { pulmonar com } \\
\text { baciloscopia }\end{array}$ & $\begin{array}{l}\text { Proporção de casos } \\
\text { de retratamento } \\
\text { com cultura }\end{array}$ & $\begin{array}{l}\text { Proporção } \\
\text { de casos } \\
\text { curados }\end{array}$ & $\begin{array}{c}\text { Proporção } \\
\text { de casos } \\
\text { curados com } \\
\text { comprovação } \\
\text { bacteriológica }\end{array}$ & $\begin{array}{l}\text { Proporção } \\
\text { de casos com } \\
\text { abandono }\end{array}$ & $\begin{array}{c}\text { Proporção } \\
\text { de casos com } \\
\text { óbito }\end{array}$ \\
\hline & $(\%)$ & $(\%)$ & $(\%)$ & $(\%)$ & $(\%)$ & $(\%)$ \\
\hline & \multicolumn{5}{|c|}{ Mediana } & \\
\hline Bom & $93,8^{\mathrm{a}}$ & $85,4^{\mathrm{a}}$ & $87,4^{\mathrm{a}}$ & $87,4^{\mathrm{a}}$ & $-{ }^{\mathrm{a}}$ & $4,9^{\mathrm{a}}$ \\
\hline Intermediário & $82,0^{\mathrm{ab}}$ & $72,2^{\mathrm{ab}}$ & $80,5^{\mathrm{ab}}$ & $81,7^{\mathrm{ab}}$ & $3,7^{\mathrm{ab}}$ & $7,5^{\mathrm{ab}}$ \\
\hline Ruim & $68,4^{b}$ & $53,8^{\mathrm{b}}$ & $74,1^{b}$ & $76,6^{b}$ & $9,5^{b}$ & $12,5^{b}$ \\
\hline
\end{tabular}

Continuação da Tabela 4

\begin{tabular}{|c|c|c|c|c|c|c|}
\hline \multirow[t]{2}{*}{ Classificação dos indicadores } & $\begin{array}{c}\text { Proporção } \\
\text { de casos com } \\
\text { tratamento } \\
\text { diretamente } \\
\text { observado (TDO) }\end{array}$ & $\begin{array}{c}\text { Proporção } \\
\text { decasosde } \\
\text { retratamento }\end{array}$ & $\begin{array}{c}\text { Proporção de casos } \\
\text { com encerramento } \\
\text { informado }\end{array}$ & $\begin{array}{l}\text { Proporção } \\
\text { de contatos } \\
\text { examinados }\end{array}$ & $\begin{array}{c}\text { Proporção de } \\
\text { casos testados } \\
\text { para o HIV* }\end{array}$ & $\begin{array}{l}\text { Escore } \\
\text { geral** }^{*}\end{array}$ \\
\hline & $(\%)$ & $(\%)$ & $(\%)$ & (\%) & $(\%)$ & $(\%)$ \\
\hline & \multicolumn{6}{|c|}{ Mediana } \\
\hline Bom & $45,0^{a}$ & $96,1^{a}$ & $50,5^{a}$ & $6,2^{\mathrm{a}}$ & $100,0^{\mathrm{a}}$ & $17,5^{\mathrm{a}}$ \\
\hline Intermediário & $20,3^{\mathrm{ab}}$ & $90,5^{\mathrm{ab}}$ & $35,2^{\mathrm{ab}}$ & $8,9^{\mathrm{ab}}$ & $98,8^{\mathrm{b}}$ & $23,0^{\mathrm{ab}}$ \\
\hline Ruim & $3,6^{b}$ & $82,4^{b}$ & $-b$ & $16,1^{\mathrm{b}}$ & - & $28,5^{b}$ \\
\hline
\end{tabular}

* Vírus da Imunodeficiência Humana

** Escore bom $=25$ a 33 pontos; Escore intermediário $=21$ a 24 pontos; Escore ruim $=11$ a 19 pontos

Notas:

Todas as diferenças foram estatisticamente significativas conforme 0 teste Kruskal Wallis $(p<0,001)$

As letras 'a,' $b$ ' e'ab' foram usadas para representar os resultados do teste de Dunn, que compara os grupos dois a dois. As letras diferentes ('a' e 'b') mostram diferenças significativas entre os grupos comparados e as letras que se repetem (' $a{ }^{\prime} e^{\prime} a b^{\prime} 0 u^{\prime} b^{\prime} e^{\prime} a b^{\prime}$ ) mostram que as diferenças não foram significativas segundo 0 teste de Dunn.

Tabela 5 - Distribuição das Regiões de Saúde segundo os escores de risco epidemiológico e de desempenho e 0 índice de gravidade do programa de controle da tuberculose no estado de Santa Catarina, 2003 a 2010

\begin{tabular}{lccc}
\hline Regiões de Saúde & Escore de risco & Escore de desempenho & Índice de gravidade \\
\hline Balneário Camboriú & 11 & 17 & 4,02 \\
Grande Florianópolis & 11 & 17 & 4,02 \\
Canoinhas & 9 & 14 & 4,06 \\
Itajaí & 12 & 19 & 4,19 \\
Tubarão & 10 & 19 & 5,39 \\
Lages & 8 & 18 & 6,32 \\
Brusque & 9 & 21 & 6,50 \\
Joinville & 11 & 26 & 6,56 \\
Criciúma & 11 & 28 & 6,91 \\
Videira & 8 & 21 & 7,04 \\
Jaraguá do Sul & 8 & 23 & 7,42 \\
Mafra & 8 & 23 & 7,42 \\
Araranguá & 8 & 25 & 7,74 \\
Xanxerê & 7 & 24 & 8,06 \\
Joaçaba & 6 & 21 & 8,13 \\
Blumenau & 8 & 31 & 8,44 \\
Rio do Sul & 5 & 22 & 8,79 \\
Timbó & 6 & 29 & 9,02 \\
Concórdia & 4 & 23 & 9,40 \\
São Miguel do 0este & 4 & 26 & 9,63 \\
Chapecó & 4 & 32 & 9,95 \\
\hline
\end{tabular}


Destacaram-se algumas Regiões de Saúde com risco baixo e desempenho bom, como São Miguel do Oeste, Chapecó e Timbó. Nestas Regiões, a ocorrência da doença não é muito elevada, possivelmente pelo fato de se encontrarem em uma fase anterior da endemia tuberculosa, diferentemente de outras Regiões com cidades mais antigas e urbanização mais maciça. As demais Regiões apresentaram comportamento mais homogêneo, (i) sob risco baixo e com desempenho intermediário ou (ii) sob risco intermediário e com desempenho bom, o que as coloca como que equiparadas quanto ao estado ou fase de evolução da endemia tuberculosa.

Do ponto de vista do risco epidemiológico, observou-se que a ocorrência da tuberculose em Santa Catarina tende a se concentrar nos municípios litorâneos ou próximos ao litoral, onde a densidade demográfica e o grau de urbanização são maiores. Notou-se, ainda, que o desempenho nas medidas de controle da doença nem sempre acompanha as necessidades de saúde: em algumas Regiões, o desempenho é justamente pior onde o risco é maior, havendo uma diferença bastante significativa entre as Regiões quanto ao alcance das metas do programa.

As taxas de incidência apresentaram variações da ordem de 15 a 19 vezes, e a variação da mortalidade foi de cerca de 9 vezes entre as Regiões. Sabe-se que a incidência da tuberculose guarda íntima relação com o número de casos pulmonares positivos e que, para haver uma inversão na evolução da epidemia tuberculosa em determinado local, é necessário que se identifique um mínimo de $70 \%$ dos pacientes pulmonares positivos na comunidade e destes, pelo menos $85 \%$ sejam curados. ${ }^{11,12}$ Esta recomendação foi adotada pela OMS e pelo Ministério da Saúde no Plano Nacional de Controle da Tuberculose - PNCT. ${ }^{5}$

A taxa de cura dos pulmonares positivos, a identificação da doença na comunidade mediante a baciloscopia de escarro - esta última, representada neste trabalho pela proporção de casos novos de tuberculose que realizaram baciloscopia - e 0 percentual de contatos examinados entre os identificados refletem o esforço da vigilância, em algumas Regiões de Saúde, para atingir as metas definidas. A taxa de cura atingiu a meta de $85 \%$ em apenas cinco Regiões, enquanto a proporção de contatos examinados entre os identificados (meta de 100\%) não foi alcançada por qualquer das 21 Regiões de Saúde. Embora o tratamento da tuberculose tenha uma eficácia teórica de 95\%, sua efetividade varia bastante, de acordo com o local, e está em torno de $75 \%$ (variação de 50 a 90\%). ${ }^{13}$ Nesse sentido, estudo realizado em três países da África subsaariana constatou que o tratamento da tuberculose com 0 esquema de curta duração é uma das intervenções de saúde mais custoefetivas..$^{14}$ Outro estudo, realizado em diferentes locais da África, Ásia e América Latina, com o objetivo de avaliar a eficácia e a segurança do tratamento com dose fixa combinada, comparando-o com o de tomadas separadas, teve como resultado favorável $93,9 \%$ no primeiro grupo e 94,6\% no segundo grupo ${ }^{15}$ Contudo, seus autores ressaltam: para se atingir essas taxas, é necessário que o tratamento tenha boa adesão dos pacientes.

No Brasil e no estado de Santa Catarina, não se tem alcançado o mínimo preconizado de cura de $85 \%$ para que ocorra declínio sustentado na incidência anual da doença. Avaliação da OMS, datada de 2005, apresenta 0 Brasil com a nona pior taxa de cura (81\%) entre os 22 países que concentram a maior carga da tuberculose, e com uma cobertura populacional de seus serviços de saúde encarregados do tratamento diretamente observado - TD0 - de 68\%, a segunda pior. ${ }^{16} \mathrm{~A}$ taxa de cura dos pacientes pulmonares positivos no Brasil foi de 70,3\% em 2010; em Santa Catarina, a mediana dessa proporção para o período considerado neste estudo foi de 78,6\%, aproximando-se mais dos achados relatados por Conde e colaboradores. ${ }^{17}$ Proporção de abandono, proporção de encerramento por óbito e proporção de retratamento correspondem à adesão dos pacientes ao tratamento.

Os valores porcentuais de pacientes em retratamento que realizaram cultura e de casos com encerramento em tempo oportuno são indicativos do esforço dos gestores e técnicos do programa para corrigir os problemas relativos à falta de adesão. A baixa adesão ao tratamento é um dos mais graves problemas a serem enfrentado pelos programas de tuberculose, em seus diferentes níveis, porque favorece 0 abandono, recidivas, emergência de resistência ao esquema antimicobacteriano, além do aumento da falência e óbito. ${ }^{17}$ Trata-se de uma situação que compromete - gravemente - a efetividade do tratamento e implica, como consequência, uma baixa taxa de cura. 
0 TDO é uma intervenção recomendada pela OMS desde 1993, tendo sido incorporado pelo PNCT desde 1998. O Ministério da Saúde tornou obrigatória a adoção da estratégia após a descentralização da atenção para os municípios, ao menos para os pacientes pulmonares positivos afetados por comorbidades. Trata-se de uma intervenção com eficácia comprovada, seja no aumento da taxa de cura, seja no número de vidas salvas. ${ }^{16,18}$ Isto foi verificado, principalmente, em experiências exitosas de países com grande incidência de tuberculose, extensa área territorial e maior contingente populacional. Caso da China, onde, de 1991 a 1995, a adoção da estratégia de TD0 permitiu atingir 90\% da população-alvo, alcançando mais de $90 \%$ de cura dos pulmonares positivos. ${ }^{19} \mathrm{E}$ da Índia: decorridos oito anos desde a implantação do programa naquele país em 1993, 40\% da população fora atingida, com taxas de cura acima de $80 \%{ }^{20}$

No Brasil e em Santa Catarina, diferentes experiências demonstram que a estratégia de TDO melhora a efetividade do tratamento. Segundo dados da Secretaria de Estado da Saúde de Santa Catarina, nos anos de 2010 e 2011, entre os pacientes sob TDO, a taxa de cura foi de $80 \%$ e a taxa de abandono, de $7 \%$; nos pacientes sob tratamento autoadministrado, observou-se 77\% de cura e 9\% de abandono. ${ }^{21} \mathrm{Tra}-$ balho realizado em Belém-PA, envolvendo 588 pacientes com tuberculose entre 2004 e 2008, mostrou melhores resultados de cura e abandono quando se utilizou o TDO, concluindo-se ser esta uma estratégia de extrema importância para alcançar menor taxa de abandono e aumentar a taxa de cura. ${ }^{22}$ Outro estudo, com o propósito de verificar adesão ao tratamento da tuberculose em uma área com população de baixa renda e alta carga de tuberculose em Carapicuíba, Grande São Paulo, concluiu que a estratégia TDO, além de poder se realizar com sucesso em unidades básicas de saúde, é mais efetiva que o tratamento autoadministrado: no município, observou-se conclusão do tratamento em 91,6\% dos pacientes sob TDO e em $85,5 \%$ dos que se submeteram ao tratamento autoadministrado. ${ }^{23}$

A qualidade do TDO realizado também é essencial para maior adesão do paciente e melhor rendimento do tratamento, conforme aponta estudo realizado em Vitória-ES, comparando dois grupos de pacientes, um (i) sob TDO tradicional, em que o paciente é medicado por agente de saúde, e outro grupo (ii) sob TDO monitorado por tutor ou provedor treinado, normalmente um membro da família. ${ }^{24}$ No primeiro grupo, $83 \%$ completaram o tratamento ou foram curados, enquanto no segundo, $98 \%$ dos pacientes completaram o tratamento ou foram curados. Os autores ressaltam que empenho, dedicação, envolvimento da comunidade ou círculo familiar são fundamentais na adesão e recuperação do paciente.

Com a disseminação da Estratégia Saúde da Família, esperava-se um incremento significativo no tratamento supervisionado. Entretanto, estudos sobre a atenção básica mostraram $89,9 \%$ dos pacientes atendidos na modalidade de tratamento autoadministrado, revelando que o tratamento supervisionado foi pouco incorporado. ${ }^{25}$

Apesar de ser disponibilizado gratuitamente, o tratamento representa um custo econômico para 0 paciente, tanto pelo custo do deslocamento como pela perda do turno de trabalho por ocasião da consulta. ${ }^{25}$ Situações como estas seriam corrigidas pela instituição universal do tratamento diretamente observado - TDO -, como determina o Ministério da Saúde.

Outros estudos apontam a importância do trabalho de equipes especializadas e da boa comunicação para estabelecer um vínculo adequado com os pacientes de tuberculose, favorecendo sua adesão ao tratamento. ${ }^{26}$ Mais aspectos capazes de influenciar negativamente no desempenho de programas de controle da tuberculose, tais como falta de recursos materiais e humanos, equipes mal dimensionadas e contexto sociocultural e econômico dos pacientes, exigem permanente qualificação gerencial, organizacional e técnico-assistencial dos profissionais de saúde. ${ }^{27}$

A testagem para 0 HIV e a possibilidade de coinfecção TB/HIV evocam outra dimensão da epidemiologia da tuberculose e um especial desafio aos municípios para o enfrentamento desse grave aspecto da doença. ${ }^{9}$ A coinfecção TB/HIV apresenta uma dinâmica própria, alavanca a virulência de ambas as doenças de forma a aumentar a letalidade, $o$ abandono pelo paciente e a resistência de seu organismo aos medicamentos antimicobacterianos., ${ }^{9,2}$ Estudo realizado em municípios com alta incidência de aids, predominantemente no sudoeste e sul do Brasil, encontrou maior gravidade da situação epidemiológica da tuberculose, de modo semelhante aos achados deste estudo. ${ }^{29}$

Se 0 óbito por tuberculose fosse uma situação excepcional - como deveria -, sua ocorrência 
refletiria problemas com o diagnóstico e o tratamento. A vigilância de óbitos por tuberculose permite aumentar a completitude dos sistemas de informações, diminuir a subnotificação e o número de casos não encerrados, supervisionar a vigilância epidemiológica realizada pelas unidades de saúde e a qualidade do preenchimento das declarações de óbito (D0); além de buscar contatos não avaliados pela equipe de saúde, como foi observado em estudo sobre óbitos por tuberculose no estado do Rio de Janeiro, cujos resultados apontaram para diagnóstico tardio, subnotificação e elevada associação com comorbidades, tabagismo, abuso de álcool e baixa testagem para o HIV. ${ }^{30}$

Uma limitação do presente estudo consistiu na utilização de dados secundários, extraídos do Sistema de Informação de Agravos de Notificação (Sinan), com possíveis imprecisões e inconsistências próprias do sistema, caso da subnotificação. Entretanto, a revisão realizada pelos órgãos estaduais de vigilância epidemiológica, previamente à realização deste estudo, minimizou tal situação. Outra limitação ao desenvolvimento do estudo, característica de estudos ecológicos, consiste na chamada falácia ecológica, para a qual os resultados encontrados em uma escala regional

\section{Referências}

1. World Health Organization. Tuberculosis: fact sheet $\mathrm{n}^{\circ} 104$ [Internet] Geneva: World Health Organization; 2013 [cited 2013 Oct 23]. Available from: http://www. who.int/mediacentre/factsheets/fs104/en

2. Ministério da Saúde (BR). Programa Nacional de Controle da Tuberculose. Tuberculose no Brasil e no mundo [Internet]. 2013 [citado 2013 mar 26]. Disponível em: http://portal.saude.gov.br/portal/ saude/profissional/visualizar_texto.cfm?idtxt=31109

3. World Health Organization. Global tuberculosis report 2013 [Internet]. Geneva: World Health Organization; 2013 [cited 2013 Nov 13]. Available from: http://apps.who.int/iris/ bitstream/10665/91355/1/9789241564656_eng.pdf

4. Ruffino-Netto A. Tuberculose: a calamidade negligenciada. Rev Soc Bras Med Trop. 2002 janfev;35(1):51-8.

5. Ministério da Saúde (BR). Secretaria de Vigilância em Saúde. Departamento de Vigilância Epidemiológica. Manual de recomendações para o controle da não traduzem, necessariamente, os resultados dos municípios circunscritos a ela quando considerados de maneira isolada.

Finalmente, espera-se que os achados do presente estudo subsidiem os gestores da vigilância epidemiológica e da atenção básica na tomada de decisões conjuntas, buscando melhorar os processos de trabalho das equipes de saúde, aprimorar o desempenho dos programas municipais de controle e, como consequência, reduzir o risco epidemiológico representado pela tuberculose.

\section{Agradecimentos}

À equipe da DIVE/SES-Santa Catarina, em especial, à coordenação do Programa Estadual de Tuberculose pelo apoio na coleta e compreensão dos dados.

\section{Contribuição dos autores}

Mendonça SA e Franco SC conceberam e delinearam o estudo, redação e revisão crítica do conteúdo intelectual. Todos os autores aprovaram a versão final do manuscrito e declaram serem responsáveis por todos os aspectos do trabalho, garantindo sua precisão e integridade.

tuberculose no Brasil [Internet]. Brasília: Ministério da Saúde; 2011 [citado 2013 ago 8]. Disponível em: http://www.cve.saude.sp.gov.br/htm/TB/mat_tec/ manuais/MS11_Manual_Recom.pdf

6. Braga JU. Vigilância epidemiológica e o sistema de informação da tuberculose no Brasil, 2001-2003. Rev Saude Publica. 2007;41 supl 1:77-88.

7. Secretaria de Estado da Saúde (Santa Catarina). Superintendência de Vigilância em Saúde. Diretoria de Vigilância Epidemiológica. Situação da tuberculose em Santa Catarina - 2008 [Internet]. Florianópolis: DIVE; 2008 [citado 2012 jul 14]. Disponível em: http://www.dive.sc.gov.br/ conteudos/agravos/publicacoes/Dados_ Estatisticos.pdf

8. Secretaria de Estado da Saúde (Santa Catarina). Plano Diretor de Regionalização: PDR 2008 [Internet]. Florianópolis: Secretaria de Estado da Saúde; 2008 [citado 2013 out 27]. Disponível em: http://www. saude.sc.gov.br/geral/planos/PDR/PDR\%202008\%20 -\%20Aprovado.pdf 
9. Getahun H, Kittikraisak W, Heilig CM, Corbett EL, Ayles H, Cain KP, et al. Development of a standardized screening rule for tuberculosis in people living with HIV in resource-constrained settings: individual participant data meta-analysis of observacional studies. PLoS Med. 2011 Jan;8(1):e1000391.

10. Ministério da Saúde (BR). Secretaria de Vigilância em Saúde. Especial tuberculose. Bol Epidemiol [Internet]. 2012 mar;43(esp):1-12 [citado 2013 jun 16]. Disponível em: http://portalsaude.saude. gov.br/images/pdf/2014/julho/23/BE-2012-43-Mar-o---Especial-Tuberculose.pdf

11. Augusto CJ, Carvalho WS, Gonçalves AD, Ceccato MGB, Miranda SS. Características da tuberculose no estado de Minas Gerais entre 2002 e 2009. J Bras Pneumol. 2013 mai-jun;39(3):357-64.

12. Styblo K. The relationship between the risk of tuberculous infection and the risk of developing infectious tuberculosis. Bull Int Union Tuberc Lung Dis. 1985;60:117-9.

13. Ministério da Saúde (BR). Fundação Nacional de Saúde. Centro de Referência Professor Hélio Fraga. Sociedade Brasileira de Pneumologia e Tisiologia. Controle da tuberculose: uma proposta de integração ensino-serviço. 5. ed. Rio de Janeiro: FUNASA; 2002.

14. Murray CJL, Dejonghe E, Chum HJ. Cost effectiveness of chemotherapy for pulmonary tuberculosis in three sub-Saharan African countries. Lancet. 1991 Nov;338(8778):1305-8.

15. Lienhardt C, Cook SV, Burgos M, Yorke-Edwards V, Rigouts L, Anyo G, et al. Efficacy and safety of a 4-drug fixed-dose combination regimen compared with separate drugs for treatment of pulmonary tuberculosis: the Study C randomized controlled trial. JAMA. 2011 Apr;305(14):1415-23.

16. Barreira D, Grangeiro A. Avaliação das estratégias de controle da tuberculose no Brasil. Rev Saude Publica. 2007 set;41 supl 1:4-8.

17. Conde M, Fiterman J, Lima M, organizadores. Tuberculose. Rio de Janeiro: Guanabara Koogan; 2011.

18. Kochi A. Tuberculosis control: is DOTS the health breakthrough of the 1990s? World Health Forum. 1997;18(3-4):225-32.
19. Xianyi C, Fengzeng Z, Hongjin D, Liya W, Lixia W, Xin D, et al. The DOTS strategy in China: results and lessons after 10 years. Bull World Health Organ. 2002 Jun;80(6):430-6.

20. Khatri GR, Frieden TR. Controlling tuberculosis in India. N Engl J Med. 2002 Oct;347(18):1420-5.

21. Secretaria do Estado da Saúde (Santa Catarina). Superintendência de Vigilância em Saúde. Diretoria de Vigilância Epidemiológica. Informativo epidemiológico, tuberculose no estado de Santa Catarina, 2013 [Internet]. Florianópolis DIVE; 2013 [citado 2013 nov 17]. Disponível em: http://www. dive.sc.gov.br/conteudos/agravos/Tuberculose/ Tuberculose_SC.pdf

22. Paz LNF, Ohnishi MDO, Barbagelata CM, Bastos FD, Oliveira III JAF, Parente IC. Effectiveness of tuberculosis treatment. J Bras Pneumol. $2012 \mathrm{Jul}$ Aug;38(4):503-10.

23. Vieira AA, Ribeiro SA. Adesão ao tratamento da tuberculose após a instituição da estratégia de tratamento supervisionado no município de Carapicuíba, Grande São Paulo. J Bras Pneumol. 2011 mar-abr;37(2):223-31.

24. Prado TN, Wada N, Guidoni LM, Golub JE, Dietze R, Maciel ELN. Cost-effectiveness of community health worker versus homebased guardians for directly observed treatment of tuberculosis in Vitória, Espírito Santo State, Brazil. Cad Saude Publica. 2011 May;27(5):944-52.

25. Figueiredo TMRM, Villa TCS, Scatena LM, Gonzales RIC, Ruffino-Netto A, Nogueira JA, et al. Desempenho da atenção básica no controle da tuberculose. Rev Saude Publica. 2009 out;43(5):825-31.

26. Brunello ME, Cerqueira DF, Pinto IC, Arcêncio RA, Gonzales RI, Villa TC, et al. Interaction between patient and health care professionals in the management of tuberculosis. Acta Paul Enferm. 2009;22(2):176-82.

27. Arakawa T, Arcencio RA, Scatolin BE, Scatena LM, Ruffino-Netto A, Villa TCS. Acessibilidade ao tratamento de tuberculose: avaliação de desempenho de serviços de saúde. Rev Latino-Am Enferm. 2011 jul-ago;19(4):994-1002.

28. Glaziou P, Falzon D, Floyd K, Raviglione M. Global Epidemiology of Tuberculosis. Semin Respir Crit Care Med. 2013 Feb;34(1):3-16. 
29. Gonçalves MJF, Penna MLF. Morbidade por tuberculose e desempenho do programa de controle de controle em municípios brasileiros, 2001-2003. Rev Saude Publica. 2007 set;41 supl 1:95-103.

30. Selig L, Belo M, Cunha AJLA, Teixeira EG, Brito R, Luna AL, et al. Óbitos atribuídos à tuberculose no
Estado do Rio de Janeiro. J Bras Pneumol. 2004 julago;30(4):327-34.

Recebido em 05/03/2014

Aprovado em 15/01/2015 